\title{
Comparing Error Estimators for Runge-Kutta Methods
}

\author{
By L. F. Shampine and H. A. Watts*
}

\begin{abstract}
A way is proposed to compare local error estimators. This is applied to the major estimators for fourth-order Runge-Kutta procedures. An estimator which leads to a production code $18 \%$ more efficient than a code using the standard one is recommended and supported by numerical tests.
\end{abstract}

1. Introduction. The design of a production code for the numerical solution of ordinary differential equations requires three major choices (at least): the method of advancing the solution one step, the method of estimating the local error incurred in one step, and the strategy for choosing the next step length. The first choice has been intensively studied in the literature of numerical analysis but the latter two have been rather neglected in spite of their great practical significance. The aim of this paper is to compare alternatives for estimating the local error. We restrict ourselves to estimators appropriate to fourth-order Runge-Kutta methods for two reasons. Production codes based on fourth-order Runge-Kutta processes are quite common and we are able to recommend improvements based on this study. Secondly, texts frequently mention how easy it is to change the step size when using Runge-Kutta procedures but they often fail to point out the associated practical disadvantages. Namely, it is rather difficult and expensive to estimate the local error and so decide when to change the step size. Furthermore, the standard estimator forces one to use a relatively crude step size strategy. It is, therefore, important to consider the reliability and efficiency of error estimating schemes.

In Section 2, we shall propose a way of comparing numerically local error estimators which seems to be of broad applicability. Section 3 describes the most useful error estimators known to the authors, which are then compared in the fourth section. On the basis of this comparison and other facts presented, we recommend a RungeKutta process using an estimator different from the standard one. To further support this recommendation, we describe in Section 5 the results of numerical tests comparing a production code using the standard estimator and the same code modified to use the estimator we suggest.

2. Comparing Methods for Estimating Local Errors. To study numerically the relative effectiveness of local error estimators, we shall need a broad class of differential equations for which we can evaluate the true solution for arbitrary initial conditions. Since the local error of Runge-Kutta (R-K) methods depends on the form of the equation as well as the solution, it is necessary to use a variety of non-

Received November 13, 1970, revised January 21, 1971.

AMS 1969 subject classifications. Primary 6561, 6580.

Key words and phrases. Local error, error estimators, Runge-Kutta methods, step size adjustment, solution of differential equations.

* This was partially supported by the U. S. Atomic Energy Commission. 
linear equations. Although the class we suggest can be treated for systems of equations, we restrict our attention to a single equation

$$
y^{\prime}(x)=f(x, y(x)), \quad y\left(x_{0}\right)=y_{0} .
$$

We suggest using the class of problems defined by those $f$ which can be represented by a convergent double power series near the point of interest $\left(x_{0}, y_{0}\right)$. Without loss of generality, we shall assume that $\left(x_{0}, y_{0}\right)=(0,0)$, so that the initial-value problem becomes

$$
y^{\prime}(x)=\sum_{i, j=0}^{\infty} a_{i j} x^{i} y^{j}=f(x, y), \quad y(0)=0 .
$$

The differential equation then has a unique solution analytic in some neighborhood of the origin and can be represented by a power series there,

$$
y(x)=\sum_{k=1}^{\infty} c_{k} x^{k}
$$

The coefficients can be derived recursively as follows: Define $c_{k, j}$ as the coefficient of $x^{k}$ in the $j$ th power of $y$,

$$
[y(x)]^{j}=\sum_{k=1}^{\infty} c_{k, j} x^{k}
$$

Noting that $c_{k, i}=0$ for $k<j$ and that $c_{k, 1} \equiv c_{k}$ we easily obtain

$$
c_{k, i+1}=\sum_{n=1}^{k-j} c_{n} c_{k-n, j} \quad \text { for } j \geqq 1 \text {. }
$$

On substituting these series in (1) and equating coefficients, we get

$$
k c_{k}=a_{k-1,0}+\sum_{i=0}^{k-2}\left(\sum_{j=1}^{k-1-i} a_{i j} c_{k-1-i, j}\right) \text { for } k \geqq 1,
$$

where we take $\sum_{0}^{-1}=0$.

For our numerical studies we, of course, truncate the series (1), (2) but we still need to know the region of convergence. Let us suppose that the $a_{i j}$ satisfy $\left|a_{i j}\right| \leqq 1$. (We shall, in fact, generate the $a_{i j}$ randomly and uniformly distributed in the interval $[-1,1]$.) The method of majorants then guarantees that the series (2) has a radius of convergence of at least $1-\exp \left(-\frac{1}{2}\right) \doteq 0.3935$ [1, pp. 282-283].

Let us now describe a way of comparing local error estimators. By definition, the actual error incurred in a single step of length $h$, say from $x=0$ to $x=h$, is

$$
\tau(h) \equiv \text { true local error }=y_{h}-y(h)
$$

where $y_{h}$ denotes the approximate solution given by the numerical procedure. For a method of order $p$, the local error is, by definition, $O\left(h^{p+1}\right)$. We further assume that for this single step

$$
\tau(h)=c h^{p+1}+a h^{p+2}+O\left(h^{p+3}\right),
$$

where $c$ and $a$ are constants (depending on $f$ but independent of $h$ ). A scheme for estimating this local error ought to be asymptotically correct; that is, the estimated local error, $\epsilon(h)$, should satisfy 


$$
\lim _{h \rightarrow 0} \frac{\epsilon(h)}{\tau(h)}=\mu,
$$

where $\mu$ is a finite constant equal to one for those problems with $c \neq 0$. We shall only consider such procedures; so let us write

$$
\epsilon(h)=c h^{p+1}+b h^{p+2}+O\left(h^{p+3}\right),
$$

where $b$ is a constant (depending on $f$, independent of $h$ ). We shall now discuss how we may compare estimators for sufficiently small $h$. For this asymptotic comparison we compute

$$
d(f) \equiv \lim _{h \rightarrow 0} \frac{\epsilon(h)-\tau(h)}{h^{p+2}}=b-a .
$$

It may seem unlikely that one would consider estimators which are not asymptotically correct. Nevertheless, in the next section, we shall mention two such estimators. An important point to be kept in mind is that one might base his error control on a quantity other than local error. For example, the global error is really the quantity of interest though this option seems almost unexplored. However, granted that one estimates local error and that his estimator is at least asymptotically correct, it seems reasonable to compare estimators by their effectiveness in estimating the next term in the asymptotic expression for the local error.

Computationally, we proceed as follows: For a given problem (1), where the $a_{i j}$ are generated randomly in the interval $[-1,1]$, we compute $\epsilon$ and $\tau$ for a sequence of $h$ values for each method being tested. This sequence consists of $h$ values decreasing to zero with the initial (largest) $h$ chosen so that the series (2) is convergent for $x=$ $\pm 2 h$. Since we approximate asymptotic results $(h \rightarrow 0)$, the series converges very rapidly. Now, for each $h$ of this sequence, we compute the ratio in (9), until successive approximations to $d(f)$ differ by less than a prescribed amount. Finally, we compute the average and maximum values of our approximations to $|d(f)|$ over an ensemble of randomly chosen problems for each method.

3. Some Error Estimating Schemes. We shall first describe two estimators which we shall not study and explain why. The Merson process [2] is used in a number of codes. However, it does not seem to be adequately appreciated that the justification of this method is restricted to equations of the form $f(x, y)=\alpha x+\beta y+\gamma$. The method is not asymptotically correct for general nonlinear equations, as the simple example $y^{\prime}(x)=x+x y(x), y(0)=0$ shows clearly. A little computation results in

$$
\tau(h)=\frac{-1}{432} h^{6}+O\left(h^{8}\right),
$$

whereas Merson's estimator gives

$$
\epsilon(h)=\frac{-1}{180} h^{4}+O\left(h^{6}\right)
$$

so that Eq. (7) fails to be satisfied. Since the method is without theoretical support for general equations, we recommend it be abandoned. It is worth comment that although asymptotically the estimate bears no useful relationship to the true error, 
codes based on it are too conservative in the choice of step size as the order of the error is estimated to be too low. Thus, they are simply inefficient.

Zonneveld [3] gives an error estimation scheme which cannot be compared using the method of the previous section, because he does not estimate the local truncation error. He estimates the last term of the Taylor expansion that has been taken into account. For a fourth-order process, this is the contribution of order $h^{4}$ which enters into the value of $y_{h}$. His numerical results seem quite satisfactory though his step size strategy may compensate for any shortcomings of the error estimator. Hull [4] reports some limited comparison with the doubling procedure we shall now describe.

Extrapolation procedures are the most familiar and widely used techniques for estimating local errors associated with Runge-Kutta methods. These compute solutions (locally) twice with different step sizes and combine the results to estimate the error in one of the values. The most common choice is to compute $y_{2 h}$ by two steps of length $h$ and $y_{2 h}^{*}$ by one step of length $2 h$. The average of the error accumulated in the two steps is then estimated by

$$
\epsilon(h)=\frac{y_{2 h}^{*}-y_{2 h}}{30}
$$

for a fourth-order R-K method. This procedure will be referred to as doubling and it seems fair to describe it as the standard estimator for R-K methods. The technique is not limited to fourth-order processes nor to one-step methods. Theoretical details and justification may be found in [5, pp. 80-82]. If the estimated error is acceptable, we expend $5 \frac{1}{2}$ function evaluations per step using any of the standard fourth-order $\mathrm{R}-\mathrm{K}$ processes. If the estimated error is unacceptable, we reduce $h$ to $h / 2$ so that some of the data computed may be reused. Then, an unacceptable error results in a loss of seven function evaluations before we recycle the computations. Although this may not be the most efficient refinement logic with respect to number of function evaluations per unit increase in $x$, such strategy seems to be universally used in production codes.

The next class of methods we shall consider may be referred to as multistage error estimates which are based on approximate quadrature and local expansions at several neighboring points. Estimates of this type may be found in [6] and again do not depend on any particular R-K process. Let us note that the error is being averaged over several steps and may be unreliable when the error is changing rapidly. A further disadvantage is that one now has the complications associated with the construction of a multistep code. We shall be concerned with four such estimates. The most accurate formula listed by Ceschino and Kuntzmann is of the form

$$
\epsilon(h)=\frac{1}{60}\left(11 y_{n+1}+27 y_{n}-27 y_{n-1}-11 y_{n-2}\right)-\frac{h}{20}\left(f_{n+1}+9 f_{n}+9 f_{n-1}+f_{n-2}\right) .
$$

One can obtain such a formula via the Hermite interpolating polynomial. Theoretical justification of its use is rather complicated and only sketched in [6, pp. 248-249].

A detailed derivation shows this formula to be more accurate (as applied to methods of order $\leqq 4$ ) when the estimated error is taken to be the error in going from $x_{n-1}$ to $x_{n}$. In the context of our numerical comparison scheme (recall we are 
interested in the error incurred in going from $x=0$ to $x=h$ ), Eq. (11) becomes

$$
\epsilon(h)=\frac{1}{60}\left(11 y_{2 h}+27 y_{h}-11 y_{-h}\right)-\frac{h}{20}\left(f_{2 h}+9 f_{h}+9 f_{0}+f_{-h}\right),
$$

using the initial condition $y_{0}=0$.

Here $y_{2 h}, y_{h}$ and $y_{-h}$ are taken to be the numerical solutions obtained from the R-K process at the points $x=2 h, h,-h$, respectively, where $y_{-h}$ results from an integration proceeding backward one step from $x=0$ and $y_{2 h}$ is the two-step solution as discussed in the doubling procedure. This way of supplying the needed data for the error formula seems best for an unbiased comparison of the various estimators. Now, utilizing some fourth-order R-K process in a numerical algorithm with (11) being applied for estimating the error over $x_{n-1}$ to $x_{n}$, we expend four function evaluations per step as long as the error is tolerable (since we can reuse $f_{n+1}$ as $f_{n}$ in the next step). If not, and our acceptability criterion calls for a reduction in step, we lose eight function evaluations and must fill in new values for $y_{n-2}$ and $f_{n-2}$ which are needed in the estimator. These would be obtained most cheaply via interpolation. An advantage over doubling in the step size strategy is that no additional penalties are incurred in reducing the step to other than $h / 2$.

Alternatively, if we wish to interpret the multistage error estimate (11) as representing the error over $x_{n}$ to $x_{n+1}$, the equation for the comparison scheme becomes

$$
\epsilon(h)=\frac{1}{60}\left(11 y_{h}-27 y_{-h}-11 y_{-2 h}\right)-\frac{h}{20}\left(f_{h}+9 f_{0}+9 f_{-h}+f_{-2 h}\right) .
$$

As before, the values $y_{-h}$ and $y_{-2 h}$ are computed solutions obtained via the R-K process by backward integration of one and two steps, respectively. Again, this way of supplying the required values is merely for our comparison purposes, the intent being to approximate the solution errors introduced in a normal application of the estimator (11). The advantage of such a numerical algorithm is that one obtains an estimate of the error incurred over the single step in which the solution is being advanced, thereby resulting in a loss of only four function evaluations when the current step is rejected. Again, let us assume that the missing values required for the estimator are to be supplied by interpolation. We find that reducing the step to $h / 2$ requires new values for $y_{n-1}$ and $f_{n-1}$ whereas reduction to other than $h / 2$ requires the additional values $y_{n-2}$ and $f_{n-2}$.

We initially included two other multistage formulas in our comparisons. These were

$$
\epsilon(h)=\frac{1}{90}\left(33 y_{n+1}+24 y_{n}-57 y_{n-1}\right)-\frac{h}{90}\left(10 f_{n+1}+57 f_{n}+24 f_{n-1}-f_{n-2}\right)
$$

and

$$
\epsilon(h)=\frac{1}{30}\left(y_{n+1}+18 y_{n}-9 y_{n-1}-10 y_{n-2}\right)-\frac{h}{30}\left(9 f_{n}+18 f_{n-1}+3 f_{n-2}\right),
$$

formulas $V^{*}(a)$ and $V$ of [6, p. 250], respectively. (Formula (15) was first given by Morel [7].) Although Morel's estimator was more accurate than (14), they were both found to be considerably less accurate on the average than were (12) and (13). Because of this and the fact that they offer no other significant advantage (though use 
of Eq. (15) in estimating the error over $x_{n}$ to $x_{n+1}$ does result in a loss of only three function evaluations if refinement occurs), these estimators were dropped from our final tests.

Our next estimator is not as well known as the previous methods. England [8] has recently given some very efficient processes which use quantities calculated during a R-K step in an "internal" error estimating formula. The particular scheme of most appeal to us is given in Eqs. (8) and (9) of his paper and is completely comparable to the technique of doubling.

To get some feeling for the internal estimators, we derived a simple family for the Euler-Cauchy second-order Runge-Kutta process:

$$
\begin{aligned}
& k_{0}=h f\left(x_{0}, y_{0}\right), \\
& k_{1}=h f\left(x_{0}+h, y_{0}+k_{0}\right), \\
& y_{1}=y_{0}+\frac{1}{2}\left(k_{0}+k_{1}\right) .
\end{aligned}
$$

We begin on the second step, perform an extra function evaluation, and estimate the error in $y_{1}$ :

$$
\begin{aligned}
k_{2} & =h f\left(x_{1}, y_{1}\right), \\
k_{3} & =h f\left(x_{1}+\alpha h, y_{1}+\beta k_{2}\right), \\
r_{1} & =\delta_{0} k_{0}+\delta_{1} k_{1}+\delta_{2} k_{2}+\delta_{3} k_{3} .
\end{aligned}
$$

The parameters $\alpha, \beta, \delta_{0}, \delta_{1}, \delta_{2}, \delta_{3}$ are to be chosen so that $y\left(x_{1}\right)-y_{1}=r_{1}+O\left(h^{4}\right)$. A relatively simple expansion and equating of coefficients leads to the equations $\alpha=\beta, \delta_{0}=-\left(\delta_{1}+\delta_{2}+\delta_{3}\right)$ and

$$
\begin{aligned}
\delta_{1}+\delta_{2}+(1+\alpha) \delta_{3} & =0, \\
\delta_{2}+(2 \alpha+1) \delta_{3} & =\frac{1}{3}, \\
\delta_{1}+\delta_{2}+(1+\alpha)^{2} \delta_{3} & =-\frac{1}{6} .
\end{aligned}
$$

For given $\alpha$, there is a unique solution if and only if $\alpha \neq 0,-1$. The excluded cases are not surprising since, for them, $k_{3}$ does not provide a new function evaluation. A very desirable choice is $\alpha=1$, for then

$$
\begin{aligned}
& k_{3}=h f\left(x_{1}+h, y_{1}+k_{2}\right), \\
& y_{2}=y_{1}+\frac{1}{2}\left(k_{2}+k_{3}\right), \\
& r_{1}=\left(-k_{0}-5 k_{1}+7 k_{2}-k_{3}\right) / 12,
\end{aligned}
$$

and if $r_{1}$ is tolerable, we have $y_{2}$ immediately available and the error estimate is obtained with no extra expense.

England's scheme uses a particular fourth-order R-K procedure which calculates the first step by

$$
\begin{aligned}
& k_{0}=h f\left(x_{0}, y_{0}\right), \\
& k_{1}=h f\left(x_{0}+\frac{1}{2} h, y_{0}+\frac{1}{2} k_{0}\right), \\
& k_{2}=h f\left(x_{0}+\frac{1}{2} h, y_{0}+\frac{1}{4}\left(k_{0}+k_{1}\right)\right), \\
& k_{3}=h f\left(x_{0}+h, y_{0}-k_{1}+2 k_{2}\right), \\
& y_{1}=y_{0}+\frac{1}{6}\left(k_{0}+4 k_{2}+k_{3}\right),
\end{aligned}
$$


and starts calculating the second step in the same manner,

$$
\begin{aligned}
& k_{4}=h f\left(x_{0}+h, y_{1}\right), \\
& k_{5}=h f\left(x_{0}+\frac{3}{2} h, y_{1}+\frac{1}{2} k_{4}\right), \\
& k_{6}=h f\left(x_{0}+\frac{3}{2} h, y_{1}+\frac{1}{4}\left(k_{4}+k_{5}\right)\right) .
\end{aligned}
$$

Before completing this step, an extra function evaluation is made which allows us to estimate the local error accumulated in two steps,

$$
\text { 6) } \begin{aligned}
k_{7} & =h f\left(x_{0}+2 h, y_{0}+\frac{1}{6}\left(-k_{0}-96 k_{1}+92 k_{2}-121 k_{3}+144 k_{4}+6 k_{5}-12 k_{6}\right)\right), \\
r & =\frac{1}{90}\left(-k_{0}+4 k_{2}+17 k_{3}-23 k_{4}+4 k_{6}-k_{7}\right) .
\end{aligned}
$$

Now, if $r$ is tolerable, we complete the computation of the second step,

$$
\begin{aligned}
& k_{8}=h f\left(x_{0}+2 h, y_{1}-k_{5}+2 k_{6}\right), \\
& y_{2}=y_{1}+\frac{1}{6}\left(k_{4}+4 k_{6}+k_{8}\right),
\end{aligned}
$$

and continue as before.

If the step size of $h$ is acceptable, a total of $4 \frac{1}{2}$ function evaluations per step are made. If the step is to be rejected, we lose seven function evaluations. In this regard, let us note that England's scheme is more flexible than doubling since we can reduce $h$ by any suitable factor without penalty. This remark is quite important to the design of codes using optimally chosen step sizes.

Using the same notation as above, our error estimate over $x=0$ to $x=h$ is

$$
\epsilon(h)=\frac{1}{180}\left(k_{0}-4 k_{2}-17 k_{3}+23 k_{4}-4 k_{6}+k_{7}\right),
$$

where we substitute $x_{0}=0, y_{0}=0$ in the formulas leading to (16). Note that the estimate of the local error incurred in one step is taken to be the average of the estimated error over two steps, just as in doubling.

4. Numerical Comparisons. In order not to introduce possible extraneous effects from different $\mathrm{R}-\mathrm{K}$ processes, we have performed all comparisons using the R-K process of England's scheme. In point of fact, while one method might perform considerably better than another on a chosen equation, all the common fourth-order $\mathrm{R}-\mathrm{K}$ processes perform about the same on the average with perhaps a slight advantage given to those with "optimal" parameters. However, it seems more appropriate to our aim of comparing estimators to use the same Runge-Kutta method in all cases. The results we give are obtained from the error estimates of Eqs. (10), (12), (13) and (17).

Let us comment further about the actual numerical experiments. All computations were performed on a CDC 6600 computer using double precision in nearly all calculations. (A double-precision word constitutes approximately 29 decimal digits.) The statistics have been accumulated over a set of 500 problems in which twenty terms were carried in the series (1), (2). Final results were actually obtained by averaging the statistics from five different sets of 100 problems each. Since the approximate $d(f)$ values for the different problem sets were in good agreement and all data yielded consistent interpretations, we were satisfied that our ensemble formed a large enough sample to extract meaningful statistics. 
We chose $\left\{h=2^{-N} ; N=3,4, \cdots, 16\right\}$ for our sequence of $h$ values and accepted "convergence" of the $d(f)$ values if for two successive $h$ 's they agreed within 2.5 percent. (To collect additional data we actually forced a minimum of ten such iterations to take place.) Usually, the convergence criterion was satisfied at this stage, though about 10 percent of the problems did not produce "converged" $d(f)$ values. A detailed study of these problems indicated that the computations were being limited by the precision available so that the results could not be improved by considering smaller values of $h$. These may be described as "easy" problems in the sense that one or more of the estimators seemed to produce a $d(f)$ value which was at least an order of magnitude less than the corresponding average value obtained. In any attempt to compute asymptotic limits of differences, it is implicitly necessary to meet the convergence test before all significance is lost in the computer word. The long word length we used makes the situation infrequent but to avoid meaningless results, it was necessary to reject and replace by other random problems those problems for which significant values could not be obtained in this precision. Also, since the estimator (12) generally produced values of $d(f)$ which were smaller than the others by roughly a factor of ten, we decided to relax the acceptance criterion for it to 5 percent. The net effect of this discussion is that about 8 percent of the 500 problem ensemble were replaced by other random problems not causing any convergence difficulties. Table 1 gives the asymptotic results thus compiled. For easy recognition, we refer to estimators (10) as doubling, (12) as C-K\#1, (13) as C-K\#2, and (17) as England.

TABle 1. Asymptotic Comparisons

\begin{tabular}{lcl}
\hline & average $|d(f)|$ & maximum $|d(f)|$ \\
\hline C-K\#1 & .0024 & .016 \\
England & .019 & .11 \\
Doubling & .019 & .12 \\
C-K\#2 & .037 & .23 \\
\hline
\end{tabular}

Thus, asymptotically, the Ceschino-Kuntzmann estimator (12) is the most accurate since it obtains the best agreement with the error term of order $h^{6}$. In nearly all problem rejections due to limiting precision this formula was the major cause. That is, one could argue that the average given above is somewhat conservative relative to the other three estimators. An important conclusion is that asymptotically the doubling and England estimators have virtually identical behavior.

While we recognize that it is difficult to obtain meaningful results about nonasymptotic behavior (there is a strong problem dependence here), we feel that the following statistics collected do give the reader some feeling as to the performance of the various methods in this respect. For each $h$ and each method, we computed the relative discrepancies

$$
R(h)=\left|\frac{\tau(h)-\epsilon(h)}{\tau(h)}\right| .
$$


The averages, taken over the 500 problem ensemble, are given in Table 2 . We also show the average values of $|\tau(h) / y(h)|$ which are the relative sizes of the actual errors made in the $R-K$ step.

TABLE 2. Nonasymptotic Comparisons

\begin{tabular}{lccccc}
\hline \multicolumn{7}{c}{$N=-\log _{2} h$} \\
& 3 & 4 & 5 & 6 & 7 \\
\hline C-K\#1 & 1.4 & 24. & .11 & .16 & .01 \\
England & .98 & 31. & .27 & 1.9 & .10 \\
Doubling & .98 & 29. & .26 & 2.1 & .10 \\
C-K\#2 & 2.2 & 49. & .49 & 4.5 & .20 \\
& $8.5 * 10^{-6}$ & $5.6 * 10^{-7}$ & $3.2 * 10^{-8}$ & $1.7 * 10^{-9}$ & $1.1 * 10^{-10}$ \\
& \multicolumn{7}{c}{ average $|\tau(h) / y(h)|$} \\
\hline \multicolumn{7}{c}{}
\end{tabular}

For all smaller $h$ 's the values of $R(h)$ decrease fairly smoothly with the relative comparisons maintaining essentially the factors shown for $N=7$. While the erratic behavior of Table 2 is generally to be expected, we attempted to sort out what might be the typical situation. In a somewhat ad hoc fashion, we filtered out nine of the problems in which $R(h)$ seemed unreasonably large for the corresponding $h$. These might be termed "hard" problems. A detailed examination of these revealed that the difficulties were encountered when the true error $\tau(h)$ changed signs between successive $h$ 's. It was observed that two factors then created a rather large $R(h)$. In some instances, the change in sign was not immediately detected by $\epsilon(h)$ and usually the value of $\tau(h)$ was somewhat smaller than the expected local error. Table 3 gives the same information as Table 2 without the presence of the nine "hard" problems.

TABle 3. Nonasymptotic Comparisons

\begin{tabular}{lccccc}
\hline & \multicolumn{5}{c}{$N=-\log _{2} h$} \\
& 3 & 4 & 5 & 6 & 7 \\
\hline C-K\#1 & 1.4 & .31 & .07 & .02 & .006 \\
England & .96 & .40 & .20 & .09 & .045 \\
Doubling & .96 & .41 & .19 & .09 & .045 \\
C-K\#2 & 2.2 & .82 & .39 & .18 & .09 \\
& $8.5 * 10^{-6}$ & $5.6 * 10^{-7}$ & $3.2 * 10^{-8}$ & $1.7 * 10^{-9}$ & $1.1 * 10^{-10}$ \\
& \multicolumn{7}{c}{ average $|\tau(h) / y(h)|$} \\
\hline
\end{tabular}

The smooth trends of this data continue for all smaller $h$ 's and again we note that there is almost no distinction between doubling and England's estimator. Examination of the corresponding $|\tau / y|$ values shows that the estimators may be considered reliable only when $|\tau / y|$ is approximately $10^{-6}$ and smaller. One is tempted to extrapolate the data of Table 3 for larger values of $h$ and, therefore, larger values for 
$|\tau / y|$. If this is done, the graphs of the data lead one to suspect that the estimating procedures of doubling and England's scheme perform considerably better on a nonasymptotic basis than do the multistage estimators. Of course, our study does not substantiate this, but let us also recall that the estimators (12), (13) effectively average the errors committed in three steps compared to averaging the errors over two steps as in (10), (17). Thus, it seems plausible that large step sizes with a rapidly changing error could conceivably result in less reliable estimates from the multistage formulas.

We also monitored the ratios $\epsilon(h) / \tau(h)$ for distinguishing features of under- and over-estimations by the various methods. While these effects did separate the estimators, the similarities were clearly evident and it was felt that no important pattern emerged. Furthermore, we observed roughly symmetrical behavior in each estimator with respect to under- and over-estimation by certain predetermined amounts.

5. Conclusions and Tests of a Production Code. The results of the preceding section clearly show that the Ceschino-Kuntzmann estimator (12) is the most accurate, asymptotically. However, practical considerations appear to us to make it somewhat undesirable. The scheme partially destroys the flexibility afforded by one-step methods and requires a relatively complicated code. To fully utilize the good stability properties of Runge-Kutta schemes, it seems necessary to use "large" $h$, but the nonasymptotic behavior of this method is questionable. Nevertheless, the use of (12) in a code being designed should be considered since further study is needed. The closely related estimator (11) is not competitive on the grounds of accuracy though it is less costly.

The method of doubling and that of England are entirely comparable. Both estimate precisely the same quantity-the error over two steps-and their accuracies are essentially identical. England's scheme is substantially cheaper and is more flexible for the purposes of step adjustment. The logical structures of the doubling estimator and England's are so similar that it is an easy matter to alter a doubling code to use the England estimator. We recommend this be done.

To demonstrate the effect of altering a doubling code to use England's estimator, we modified a production code [9] in this way and ran a number of tests. The code to be modified uses the classical fourth-order R-K procedure so in fact we tested three codes. One was the production code with doubling and the classical choice of parameters. Another used doubling and England's choice of parameters, and the third used England's estimator and parameters. We attempted to modify as little code as possible when incorporating these changes. Test problems were drawn from a number of sources and were augmented with problems for which the two integration processes performed quite differently. Runs were made with three kinds of error criterionrelative, absolute, and mixed-and requested error tolerances of $10^{-k}$, for $k=$ $2,4,6,8,10$. In most cases, average and maximum errors were evaluated on ten equally spaced points in the interval of integration.

Full details of the tests and the results are presented in the report [10]. The data rather uniformly show that all three codes performed with the same accuracy on the average and the England estimator required approximately 18 percent fewer function evaluations than the codes using doubling. This verifies experimentally what the function counts of Section 3 would lead us to expect. 
We feel a strong case has been made for altering a doubling code to use England's estimator. While it appears that going to a Ceschino-Kuntzmann estimator might lead to still more efficient codes, we do not know of any numerical evidence to substantiate this. We have not yet sought to make a comparison of this kind.

Mathematics Department

University of New Mexico

Albuquerque, New Mexico 87106

Division 9422

Sandia Laboratory

Albuquerque, New Mexico 87115

1. E. L. INCE, Ordinary Differential Equations, Dover, New York, 1956.

2. J. ChRISTIANSEN, "Numerical solution of ordinary simultaneous differential equations of the 1st order using a method for automatic step change," Numer. Math., v. 14, 1970, pp. 317-324.

3. J. A. Zonneveld, Automatic Numerical Integration, Math. Centre Tracts, no. 8, Mathematische Centrum, Amsterdam, 1964. MR 30 \#1612.

4. T. E. HULL, The Numerical Integration of Ordinary Differential Equations, Proc. IFIP Congress 68, North-Holland, Amsterdam, 1968, pp. 131-144.

5. P. Henrici, Discrete Variable Methods In Ordinary Differential Equations, Wiley, New York, 1962. MR 24 \# B1772.

6. F. Ceschino \& J. Kuntzmann, Numerical Solution of Initial Value Problems, Dunod, Paris, 1963; English transl., Prentice-Hall, Englewood Cliffs, N.J., 1966. MR 33 \#3465.

7. H. Morel, "Evaluation de l'erreur sur un pas dans la méthode de Runge-Kutta," C. R. Acad. Sci Paris, v. 243, 1956, pp. 1999-2002. MR 18, 603.

8. R. ENGLAND, "Error estimates for Runge-Kutta type solutions to systems of ordinary differential equations," Comput. J., v. 12, 1969/70, pp. 166-170. MR 39 \#3708.

9. R. E. JONES, RUNKUT-Runge Kutta Integrator of Systems of First Order Ordinary Differential Equations, Report \#SC-M-70-724, Sandia Laboratories, Albuquerque, New Mexico, 1970.

10. L. F. Shampine \& H. A. WatTs, Efficient Runge-Kutta Codes, Report \#SC-RR-70-615, Sandia Laboratories, Albuquerque, New Mexico, 1970. (This report is available from the authors or Sandia Laboratory, Division 3428.) 